\title{
Evolución de la biomasa y del carbono acumulado por Quercus robur en Galicia (España)
}

\author{
Changes in biomass and carbon storage in Quercus robur trees in Galicia (Spain)
}

\author{
Esteban Gómez-García*, Fernando Pérez-Rodríguez
}

*Autor de correspondencia: VSonCloud S.L. (www.vsoncloud.com), Avenida de Castrelos 27, Portal $61^{\circ}$ Dcha. 36210 Vigo, España, tel.: +34 627060 844, esteban@vsoncloud.com

\begin{abstract}
SUMMARY
This study investigated changes in biomass and carbon stocks in Quercus robur trees in Galicia (NW Spain). Individual tree biomass equations that use diameter at breast height as the sole independent variable were developed. A system of additive biomass equations by component was fitted simultaneously. Heteroscedasticity and inherent correlations between biomass components were also taken into account in the GMM (generalized method of moments) fitting process. The number of trees per diameter class was obtained from the four national forest inventories (NFI) carried out to date. Carbon storage was estimated using the average carbon contents in the different tree components. Although pure stands of Q. robur in the study region have declined, the number of trees always increased in the period considered (1972-2009). The biomass also increased in the same period, from 12,209,663 $\mathrm{Mg}$ in 1972 to 40,333,571 Mg in 2009 (equivalent to 5,878,971 Mg and 19,415,955 Mg of carbon stocks, respectively).
\end{abstract}

Key words: pedunculate oak, carbon sequestration, national forest inventory (NFI).

\section{RESUMEN}

En este trabajo se ha estudiado la evolución de la biomasa y del carbono acumulado en los árboles de Quercus robur presentes en Galicia (noroeste de España). Se desarrollaron ecuaciones de estimación de biomasa para árbol individual, las cuales utilizan como variable independiente el diámetro normal. Se realizó un ajuste simultáneo para garantizar la aditividad, y se empleó la metodología GMM (generalized method of moments) debido a la presencia de heterocedasticidad. Para estimar la evolución de la biomasa de Q. robur en Galicia, las ecuaciones desarrolladas se aplicaron a los datos del número de árboles por clase diamétrica de los cuatro inventarios forestales nacionales (IFN) realizados hasta el momento. La concentración media de carbono en los diferentes componentes arbóreos considerados permitió estimar el carbono acumulado a partir de la biomasa. Aunque en Galicia ha disminuido la superficie de bosques puros de Q. robur, la evolución del número de árboles ha sido positiva en el periodo considerado (1972-2009). La evolución de la biomasa y del carbono acumulado por esta especie también ha sido positiva en este periodo, pasando de 12.209.663 Mg de biomasa arbórea en 1972 a 40.333.571 Mg en 2009, que se corresponde con 5.878.971 Mg y 19.415.955 Mg de carbono, respectivamente.

Palabras clave: roble, secuestro de carbono, inventario forestal nacional (IFN).

\section{INTRODUCCIÓN}

La importancia de la estimación de la biomasa forestal y del carbono acumulado se ha incrementado en los últimos años como resultado de la Convención sobre Cambio Climático y el Protocolo de Kyoto, donde se reconoce que los ecosistemas forestales pueden contribuir a mitigar el efecto invernadero inducido por el hombre. Por lo tanto, es esencial cuantificar el grado de secuestro de carbono atmosférico por parte de la biomasa arbórea (Dixon et al. 1994). Por cada kilogramo de carbono acumulado en la biomasa arbórea se capturan 3,67 kg de $\mathrm{CO}_{2}$. En comparación con las plantaciones comerciales, los bosques naturales juegan un papel importante como depósitos a largo plazo de carbono (Huet et al. 2004, Balboa-Murias et al. 2006).
Estimar la evolución de la biomasa forestal acumulada en una amplia región geográfica a medio o largo plazo está sujeto a una serie de limitaciones relacionadas con la ausencia de datos que definan con suficiente precisión el sistema; y por las alteraciones de entrada (plantaciones, colonización de nuevos terrenos, reclutamiento) y salida (talas, incendios, sequías, plagas, etc.) que sufre (Bolin y Sukumar 2000). Por este motivo, los inventarios forestales regionales o nacionales han sido ampliamente utilizados para la cuantificación a gran escala de la biomasa acumulada (por ejemplo, Grigal y Ohmann 1992, Birdsey et al. 1993, Isaev et al. 1995, Cohen et al. 1996, Gil et al. 2011). La evolución en la biomasa acumulada se extrae por diferencia entre inventarios. La mayoría de estos trabajos se fundamentan en una medida indirecta de la biomasa arbórea al relacionar esta con el volumen comercial, emplean- 
do los denominados factores de expansión de biomasa, o en inglés biomass expansion factors (BEF). Otro método es emplear ecuaciones de estimación de biomasa que utilizan variables independientes de árbol individual, como por ejemplo el diámetro y la altura; o de rodal como el área basimétrica; o una combinación de ambas (Gil et al. 2011). Algunos autores se refieren a las ecuaciones de estimación de biomasa como ecuaciones alométricas debido a que, normalmente, estas ecuaciones se desarrollan a partir de modelos alométricos. La elección del método de estimación de biomasa depende, entre otros factores, de la información que aporte el inventario que se toma como base para realizar los cálculos. El uso de ecuaciones de estimación de biomasa proporcionan estimaciones más precisas que el uso de factores de expansión de biomasa, ya que el primer método se basa en ecuaciones obtenidas a partir de muestreos regionales representativos; además, evita la fuente de error adicional introducida al emplear factores de expansión (Schoene 2002, Jalkanen et al. 2005, Gil et al. 2011, Castedo-Dorado et al. 2012).

En España, el inventario forestal nacional (IFN) aporta tanto el volumen comercial como las existencias (número de árboles) por especie y clase diamétrica. Es un inventario continuo donde la totalidad del territorio es recorrido cada 10 años. Su metodología se basa en la toma de datos de parcelas de un muestreo realizado en la superficie forestal arbolada a nivel provincial. Los datos obtenidos en los trabajos de campo, llevan un proceso que genera unos resultados que son publicados regionalmente a medida que se concluyen. El primer IFN se realizó en el periodo comprendido entre 1966-1975, el segundo entre 1986-1996 y el tercero entre 1997-2007. Actualmente se está realizando el cuarto IFN, aunque para Galicia (noroeste de España) ya está concluido y publicado. Según el IFN-4 para Galicia (MARM 2011), los bosques donde Quercus robur L. es la especie principal ocupan una superficie de 124.780 ha (el $8,8 \%$ de la superficie arbolada). Además de estas formaciones consideradas puras, la importancia de esta especie en Galicia se constata por su presencia en 248.184 ha (el $17,5 \%$ de la superficie arbolada) de formaciones mixtas (121.665 ha en mezcla con otras frondosas autóctonas, 105.002 ha en mezcla con Pinus pinaster Ait. y 21.517 ha en mezcla con Eucalyptus spp.). En la Península Ibérica (España y Portugal) se sitúa el límite suroeste de la distribución natural de $Q$. robur, la cual abarca la mayor parte de Europa y gran parte de Asia Menor y el Cáucaso (Díaz-Fernández et al. 1995).

Debido a la importancia de $Q$. robur en Galicia y tomando como referencia los datos de existencias de los IFN, los objetivos de este estudio son: (i) Desarrollar ecuaciones de estimación de biomasa de árbol individual para Q. robur en Galicia. (ii) Estimar la biomasa y el carbono acumulado en los árboles de esta especie presentes en la región, tanto en formaciones puras como mixtas; y su evolución en los diferentes IFN realizados hasta el momento.

\section{MÉTODOS}

Ecuaciones de estimación de biomasa para Q. robur en Galicia. La región de Galicia se caracteriza por un clima templado. La temperatura media anual es de entre 10 y 14 ${ }^{\circ} \mathrm{C}$, con un periodo medio libre de heladas de 10 meses. La precipitación anual varía entre 800 y 1.500 mm, con un ligero déficit hídrico en verano (40-150 mm) de una duración media de un mes. Los suelos más representativos se desarrollan sobre rocas graníticas o esquistos ácidos, tienen una textura franca o franco-arenosa y están bien drenados. Son suelos ricos en materia orgánica y de $\mathrm{pH}$ bajo (predominantemente en el intervalo de 4,0 a 4,5).

En el desarrollo de ecuaciones de estimación de biomasa para árbol individual, el diámetro debe ser la única variable independiente si se pretende aplicar estas ecuaciones a los datos del IFN. Las ecuaciones que están vigentes para la estimación de biomasa aérea de árbol individual para Q. robur en Galicia, desarrolladas por Gómez-García et al. (2013) con los mismos datos de este estudio, no se pueden utilizar en este trabajo, ya que emplean, además del diámetro, la altura total como variable independiente en la estimación de biomasa de algunos componentes. Las ecuaciones desarrolladas por Montero et al. (2005) solo emplean el diámetro normal como variable independiente, pero se ajustaron con una muestra de datos menos representativa (31 árboles cortados en Galicia, en el invierno de 2002-2003, en cuatro localizaciones próximas entre si) y no se empleó una metodología que asegure la aditividad del sistema de ecuaciones (Ruiz-Peinado et al. 2012). Además, el muestreo de biomasa fue realizado en invierno, por lo que los árboles muestreados no disponían de hojas, y esta fracción la ajustaron con valores estimados. Por lo tanto, se optó por el desarrollo de unas nuevas ecuaciones de estimación de biomasa aérea. Se emplearon los datos extraídos a partir del muestreo destructivo de 50 árboles. A los 31 árboles empleados por Montero et al. (2005) se agregaron los datos de 19 árboles cortados en el verano de 2009. Los 19 árboles cortados en el verano de 2009 tenían hojas y se localizaron en cinco lugares repartidos por los bosques de $Q$. robur en Galicia (figura 1). Se cortaron árboles de diferentes clases sociológicas (dominante, codominante, intermedio y dominado) y que no presentasen malformaciones o desarrollos anómalos motivados por su proximidad a claros o bordes de bosque o camino.

Ambos muestreos de biomasa (invierno de 2002-2003 y verano de 2009) se realizaron con la misma metodología. Después de la corta de cada árbol se dividió la biomasa en las siguientes fracciones: fuste (hasta $7 \mathrm{~cm}$ en punta delgada); ramas mayores de $7 \mathrm{~cm}$ (diámetro mínimo en punta delgada de $7 \mathrm{~cm}$ ); ramas de diámetro máximo en punta gruesa de $7 \mathrm{~cm}$ y mínimo en punta delgada de $2 \mathrm{~cm}$; por último, ramas menores de $2 \mathrm{~cm}$ (incluyendo hojas en el muestreo de 2009). Estas fracciones se pesaron en campo con una báscula de 50 g de precisión. A continuación se recogieron muestras de cada fracción para llevar a labora- 


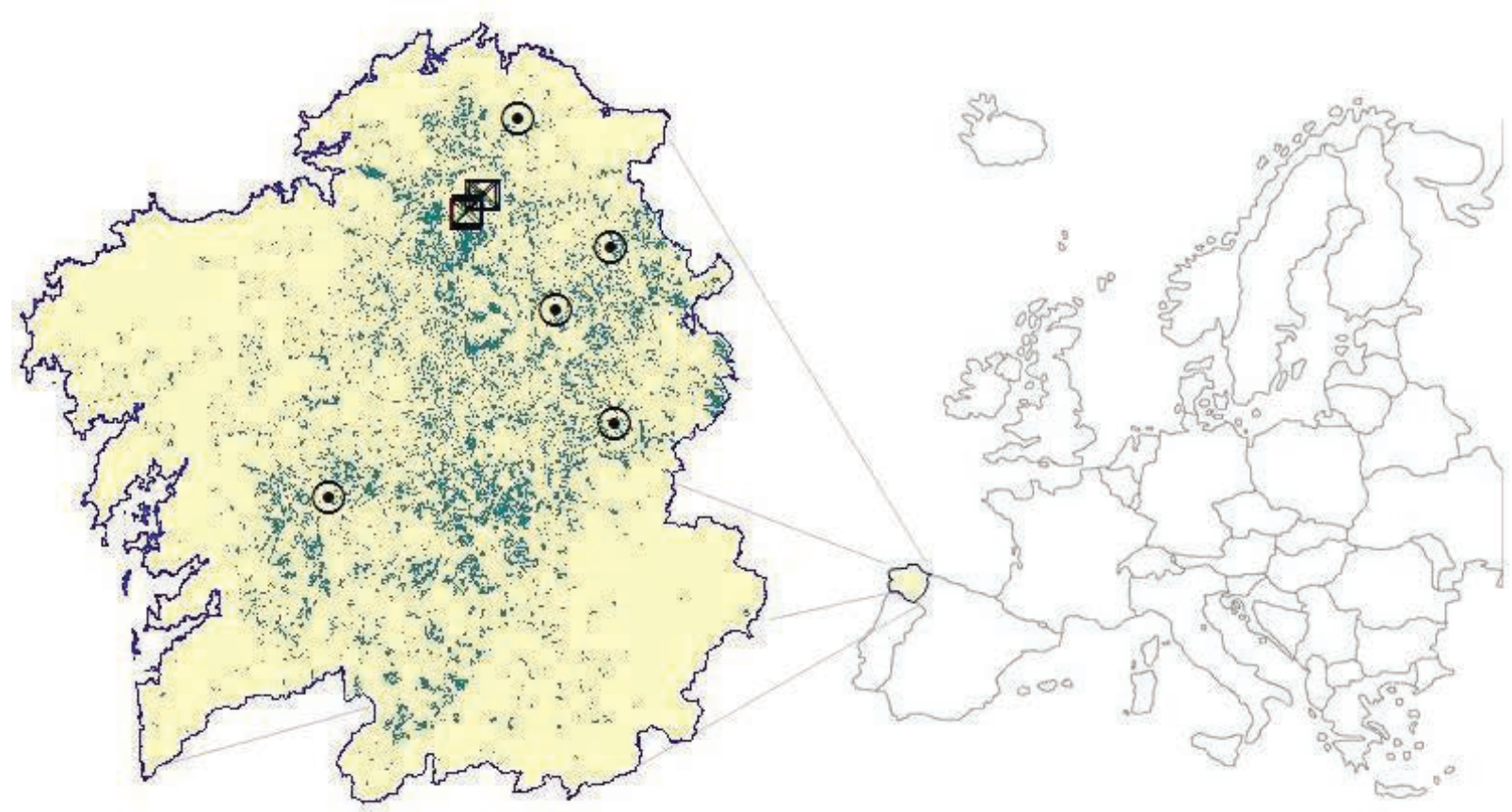

Figura 1. Localización geográfica de Galicia y lugares donde se realizaron los muestreos de biomasa para Q. robur. Los cuatro lugares donde se cortaron 31 árboles en el invierno de 2002-2003 se representan con cuadrados y los cinco lugares donde se cortaron 19 árboles en el verano de 2009 se representan con círculos. También se representan los bosques de la región donde domina esta especie.

Location of Galicia and sites where biomass sampling of $Q$. robur was carried out. The four sites where samples were obtained in winter 2002-2003 are represented by squares, and the five sites where samples were obtained in summer 2009 are represented by circles. Pure stands of this species in the region are also shown.

torio. En el fuste se cortaron 3 discos transversales de $4 \mathrm{~cm}$ de grosor (el fuste se consideró dividido en tres trozas de igual longitud y se seleccionó una rodaja en el centro de cada una de ellas). Se recogieron muestras aleatorias de ramas mayores de $7 \mathrm{~cm}$ y de ramas de 2-7 cm (aproximadamente $1,5 \mathrm{~kg}$ en cada una de estas fracciones). En las ramas menores de $2 \mathrm{~cm}$ se tomó aleatoriamente una muestra de $6 \mathrm{~kg}$ ( $2 \mathrm{~kg}$ en la parte baja de la copa, $2 \mathrm{~kg}$ en la parte media y $2 \mathrm{~kg}$ en la parte alta). Estas muestras fueron embolsadas y selladas para evitar en lo posible la pérdida de humedad. Las muestras se procesaron en laboratorio el mismo día de la corta de cada árbol. La muestra de ramas menores de $2 \mathrm{~cm}$ se dividió en ramas finas (diámetro máximo en punta gruesa de $2 \mathrm{~cm}$ y mínimo en punta delgada de $0,5 \mathrm{~cm}$ ), ramillos (diámetro máximo en punta gruesa de $0,5 \mathrm{~cm}$ ) y hojas (si las hubiese). Las muestras del fuste se dividieron en madera y corteza. La proporción, en peso verde, de estas nuevas fracciones se aplicó al árbol completo, por lo que se pasaron a considerar siete componentes: madera del fuste, corteza del fuste, ramas $>7 \mathrm{~cm}$, ramas entre $2-7 \mathrm{~cm}$, ramas entre $0,5-2 \mathrm{~cm}$, ramas $<0,5 \mathrm{~cm}$ y hojas.

El contenido en humedad de los diferentes componentes se determinó en laboratorio a partir de las muestras recogidas en campo. Una vez que las muestras fueron pesadas en verde con balanza de precisión de 0,01 g, se secaron en una estufa a $65^{\circ} \mathrm{C}$ hasta peso constante (cuando dos mediciones realizadas con una separación de 12 horas difieren menos de un $0,5 \%$ en peso) y se calculó el contenido en humedad en función de los pesos verde y seco. A partir del contenido en humedad se obtuvo el peso seco total de cada fracción, que fue el utilizado para la elaboración de las ecuaciones de biomasa. Una vez determinada la biomasa seca de los diferentes componentes de los árboles muestreados, se desarrollaron ecuaciones que relacionasen dichos pesos con el diámetro normal. El muestreo de biomasa del año 2002-2003 fue realizado en invierno, por lo tanto, los árboles muestreados no disponían de hojas. Por tal motivo, el ajuste de la ecuación de estimación de biomasa para esta fracción se realizó únicamente a partir de los datos de los 19 árboles muestreados en 2009. Se podría ajustar una única ecuación que estimase la biomasa total aérea, ya que se cumpliría con los objetivos de este estudio; pero debido a que se tenían los datos de biomasa por componentes, se prefirió desarrollar varias ecuaciones para presentarlas como un resultado complementario.

Para la estimación de la biomasa de raíces se empleó la ecuación desarrollada por Balboa-Murias et al. (2006) a partir del muestreo destructivo de 11 árboles. Aunque el número de observaciones utilizado fue reducido, es la única información disponible por el momento. En el cuadro 1 se muestran los estadísticos descriptivos de los datos empleados en el ajuste de las ecuaciones de estimación de biomasa. 
Cuadro 1. Estadísticos descriptivos del conjunto de datos empleados para el ajuste de las ecuaciones de biomasa arbórea para Q. robur en Galicia.

Descriptive statistics for the data used to develop biomass equations for Q. robur in Galicia.

\begin{tabular}{|c|c|c|c|c|c|}
\hline Variable & Observaciones & Mínimo & Máximo & Media & Desviación estándar \\
\hline \multicolumn{6}{|c|}{ Biomasa aérea } \\
\hline$d(\mathrm{~cm})$ & 50 & 5,9 & 67,5 & 27,8 & 15,4 \\
\hline$h(\mathrm{~m})$ & 50 & 5,3 & 27,6 & 16,9 & 4,7 \\
\hline$w_{m}(\mathrm{~kg})$ & 50 & 1,1 & 2297,5 & 407,3 & 524,1 \\
\hline$w_{c}(\mathrm{~kg})$ & 50 & 0,3 & 412,6 & 57,5 & 78,7 \\
\hline$w_{>7}(\mathrm{~kg})$ & 50 & 0,0 & 909,4 & 107,0 & 207,2 \\
\hline$w_{2-7}(\mathrm{~kg})$ & 50 & 4,9 & 331,0 & 72,6 & 87,6 \\
\hline$w_{05-2}(\mathrm{~kg})$ & 50 & 0,8 & 69,4 & 17,0 & 17,2 \\
\hline$w_{<05}(\mathrm{~kg})$ & 50 & 0,3 & 22,9 & 5,9 & 5,2 \\
\hline$w_{h}(\mathrm{~kg})$ & 19 & 0,4 & 20,0 & 4,3 & 5,2 \\
\hline \multicolumn{6}{|c|}{ Biomasa de raíces* } \\
\hline$d(\mathrm{~cm})$ & 11 & 14,6 & 67,5 & 34,1 & 16,1 \\
\hline$h(\mathrm{~m})$ & 11 & 11,3 & 27,6 & 18,8 & 3,9 \\
\hline$w_{r}(\mathrm{~kg})$ & 11 & 19,5 & 752,5 & 267,3 & 257,4 \\
\hline
\end{tabular}

Nota: $d$ = diámetro normal (a 1,3 m sobre el nivel del suelo), $h=$ altura total, $w_{m}=$ biomasa de madera de fuste (se considera fuste hasta $7 \mathrm{~cm}$ en punta delgada con corteza), $w_{c}=$ biomasa de corteza de fuste, $w_{>7}=$ biomasa de ramas mayores de $7 \mathrm{~cm}, w_{2-7}=$ biomasa de ramas entre 2-7 cm, $w_{<05}=$ biomasa de ramas menores de $0,5 \mathrm{~cm}, w_{h}=$ biomasa de hojas, $w_{r}=$ biomasa de raíces. *Extraído de Balboa-Murias et al. (2006).

Ajuste de modelos y criterios de selección. En un primer paso se seleccionó entre varios modelos el que presentaba mejor capacidad de ajuste para cada componente. Los modelos se ajustaron empleando el paquete estadístico SAS/STAT ${ }^{\circledR}$ (SAS Institute Inc. 2009). En un segundo paso se realizó un ajuste simultáneo para garantizar la aditividad (Kozak 1970, Parresol 1999), en el cual no se incluyó la ecuación de biomasa de hojas debido a que había menor número de observaciones para este componente. Debido a la presencia de heterocedasticidad el ajuste se realizó con la metodología GMM (generalized method of moments), con lo cual se obtuvieron estimaciones eficientes de los parámetros (Greene 2000, SAS Institute Inc. 2009). También se analizó la presencia de multicolinealidad mediante el cálculo del número de condición (Belsley 1991).

El análisis de la capacidad de ajuste de los diferentes modelos evaluados se basó en comparaciones numéricas y gráficas de los residuos. Se analizaron los gráficos de valores reales frente a valores estimados y los gráficos de residuos frente a valores estimados. Como estadísticos de ajuste se calcularon el coeficiente de determinación $\left(R^{2}-\right.$ ecuación [1]) y la raíz del error medio cuadrático (REMC - ecuación [2]).

$$
R^{2}=1-\frac{\sum_{i=1}^{i=n}\left(Y_{i}-\hat{Y_{i}}\right)^{2}}{\sum_{i=1}^{i=n}\left(Y_{i}-\bar{Y}\right)^{2}}
$$

$$
\text { REMC }=\sqrt{\frac{\sum_{i=1}^{i=n}\left(Y_{i}-\hat{Y_{i}}\right)^{2}}{n-p}}
$$

Donde

$Y_{\dot{ }}=$ valor observado de la variable dependiente.

$\hat{Y}_{i}=$ valor estimado de la variable dependiente.

$\bar{Y}=$ valor medio de la variable dependiente.

$n=$ número total de observaciones.

$p$ = número de parámetros del modelo.

Biomasa y carbono de Q. robur en Galicia y su evolución. El uso combinado de las ecuaciones de biomasa arbórea, los valores de concentración de carbono por componente y los datos de existencias recogidos en los diferentes IFN (cuadro 2) permitió determinar la cantidad de biomasa y carbono acumulado de $Q$. robur en Galicia y su evolución. El primer paso consistió en entrar en las ecuaciones de biomasa con los valores de diámetro marca de clase de la distribución diamétrica (amplitud de $5 \mathrm{~cm}$ ) recogida en los IFN. De esta forma se obtuvieron los valores modulares o promedio de biomasa para cada fracción y de biomasa total en el árbol marca de clase. Los valores de concentración de carbono se obtuvieron a partir de las muestras recogidas en el verano de 2009 (19 muestras para cada componente, menos en el de ramas $>7 \mathrm{~cm}$, donde había siete muestras). Una vez determinado el contenido de humedad de las 
Cuadro 2. Número total de árboles de Q. robur en Galicia, por clase diamétrica y en los diferentes inventarios forestales nacionales (IFN). Total number of $Q$. robur trees in each NFI in Galicia (per diameter class).

\begin{tabular}{ccccc}
\hline $\begin{array}{l}\text { Clase de diámetro } \\
(\mathrm{cm})\end{array}$ & $\begin{array}{c}\text { IFN-1 } \\
\text { (año 1972) }\end{array}$ & $\begin{array}{c}\text { IFN-2 } \\
\text { (año 1987) }\end{array}$ & $\begin{array}{c}\text { IFN-3 } \\
\text { (año 1998) }\end{array}$ & $\begin{array}{c}\text { IFN-4 } \\
\text { (año 2009) }\end{array}$ \\
\hline 5 & 59.334 .370 & 85.658 .960 & 185.176 .449 & 187.425 .000 \\
10 & 19.551 .344 & 26.116 .786 & 41.816 .712 & 65.178 .564 \\
15 & 9.195 .362 & 11.378 .874 & 18.537 .030 & 27.416 .528 \\
20 & 6.156 .407 & 7.011 .190 & 10.790 .756 & 13.697 .209 \\
25 & 4.158 .045 & 4.624 .339 & 6.863 .092 & 8.061 .977 \\
30 & 2.572 .515 & 3.672 .008 & 4.974 .735 & 5.548 .928 \\
35 & 1.550 .849 & 2.699 .597 & 3.881 .305 & 4.105 .616 \\
40 & 813.605 & 1.704 .928 & 2.467 .156 & 2.833 .540 \\
45 & 459.714 & 938.947 & 1.467 .347 & 1.788 .733 \\
50 & 187.833 & 583.669 & 1.023 .233 & 1.202 .778 \\
55 & 106.985 & 369.562 & 617.354 & 804.420 \\
60 & 41.524 & 224.134 & 322.933 & 452.611 \\
65 & 10.692 & 138.749 & 225.018 & 210.388 \\
$\geq 70$ & 22.338 & 228.089 & 397.732 & 636.683 \\
\hline
\end{tabular}

muestras se extrajo una pequeña fracción que fue molida (0,25 mg). Después se determinó la concentración de carbono mediante un autoanalizador CNS-2000 (LECO, St. Joseph, Michigan, EE.UU.). El contenido medio de carbono para cada árbol marca de clase se calculó realizando una media ponderada, con el peso de cada componente como factor de ponderación. Multiplicando la biomasa del árbol marca de clase por el número de árboles se obtuvo la biomasa total acumulada en cada clase de diámetro, y multiplicando esta por el contenido medio de carbono se obtuvo el carbono total acumulado. Por la diferencia en la biomasa y el carbono acumulados entre los distintos IFN se conoció la evolución de ambos.

\section{RESULTADOS}

Estimación de biomasa y carbono en árboles de Q. robur en Galicia. En las ecuaciones seleccionadas para la estimación de biomasa por componentes (cuadro 3) todos los parámetros estimados resultaron significativos al nivel del $5 \%$. Los gráficos de residuos frente a valores estimados mostraron un patrón aleatorio de los residuos alrededor de cero y sin tendencias anómalas. La ecuación de estimación de biomasa para ramas mayores de $7 \mathrm{~cm}$ presentó valores negativos para los árboles más pequeños, por lo tanto, cuando esta ecuación generó valores negativos $(d<18,8 \mathrm{~cm})$ se sustituyeron por el valor 0 . Esto es acorde con la inspección visual de los datos, donde se constató que a partir de aproximadamente el diámetro normal 20 $\mathrm{cm}$ los árboles empiezan a tener ramas mayores de $7 \mathrm{~cm}$. El número de condición para el ajuste simultáneo fue de
73, indicando que no hubo problemas graves de multicolinealidad (Belsley 1991). Las ecuaciones explicaron como mínimo un 78 \% de la variabilidad observada y el mejor resultado se obtuvo en la ecuación que estima la madera de fuste, con un $93 \%$.

Para simplificar la figura 2, que muestra la evolución estimada del reparto de biomasa entre los diferentes componentes a medida que el árbol aumenta en diámetro, las ramas menores de $7 \mathrm{~cm}$ se agruparon en un único componente denominado 'resto de ramas'. Los componentes que aumentan su porcentaje con respecto al total, a medida que crece el árbol, son madera de fuste, corteza de fuste y ramas mayores de $7 \mathrm{~cm}$. En contraposición los componentes que redujeron su porcentaje con respecto al total son raíces, resto de ramas y hojas. Los componentes que mayor variación presentaron al estudiar su aportación al porcentaje total son ramas $>7 \mathrm{~cm}$ y resto de ramas y los más estables fueron madera de fuste y raíces.

A partir del contenido medio de carbono en cada componente (cuadro 5) y de los valores modulares de biomasa (cuadro 4) resultó que el contenido medio de carbono para árbol total fue de 48,3\% para la clase de diámetro $5 \mathrm{~cm}$; $48,2 \%$ para las clases de diámetro 10, 15 y $20 \mathrm{~cm}$; y $48,1 \%$ para las restantes clases de diámetro consideradas $(25-70 \mathrm{~cm})$.

Evolución de la biomasa de Quercus robur en Galicia. La evolución de la biomasa arbórea y del carbono acumulado por esta especie en Galicia ha sido siempre positiva en el periodo considerado (cuadro 6 y figura 3 ). El crecimiento medio anual para toda la región en el periodo considerado 
Cuadro 3. Ecuaciones para estimar la biomasa de cada componente de Q. robur.

Equations for estimating biomass of each component of $Q$. robur.

\begin{tabular}{|c|c|c|c|c|}
\hline Componente & Ecuación & $R^{2}$ & REMC & \\
\hline Madera de fuste & $\mathrm{w}_{\mathrm{m}}=0,1151 \mathrm{~d}^{2,328}$ & 0,930 & $142 \mathrm{~kg}$ & {$[3]$} \\
\hline Corteza de fuste & $w_{c}=0,007933 d^{2,534}$ & 0,875 & $28,4 \mathrm{~kg}$ & [4] \\
\hline Ramas $>7 \mathrm{~cm}$ & $\begin{array}{l}W_{>7}=0 \text { si } d<18,8 \mathrm{~cm}, \text { si no } \\
W_{>7}=-54,96+0,1571 d^{2}\end{array}$ & 0,828 & $86,9 \mathrm{~kg}$ & {$[5]$} \\
\hline Ramas 2-7 cm & $w_{2-7}=0,06939 d^{2}$ & 0,870 & $31,9 \mathrm{~kg}$ & {$[6]$} \\
\hline Ramas 0,5-2 cm & $w_{05-2}=0,06535 d^{1,628}$ & 0,810 & $7,64 \mathrm{~kg}$ & {$[7]$} \\
\hline Ramas $<0,5 \mathrm{~cm}$ & $w_{<05}=0,03641 d^{1,502}$ & 0,815 & $2,26 \mathrm{~kg}$ & {$[8]$} \\
\hline Hojas & $w_{h}=0,01369 d^{2}$ & 0,778 & $2,53 \mathrm{~kg}$ & {$[9]$} \\
\hline Raíces* & $w_{r}=0,0851 d^{2,151}$ & 0,783 & no disponible & [10] \\
\hline
\end{tabular}

Nota: las variables están descritas en el cuadro 1. * Desarrollada por Balboa-Murias et al. (2006).

Cuadro 4. Valores modulares de biomasa (kg) en el árbol marca de clase para Q. robur en Galicia. Biomass values $(\mathrm{kg})$ for the mean tree in each diameter class for Q. robur in Galicia.

\begin{tabular}{|c|c|c|c|c|c|c|c|c|c|c|}
\hline \multirow{3}{*}{$\begin{array}{c}\text { Clase de } \\
\text { diámetro } \\
(\mathrm{cm})\end{array}$} & \multicolumn{8}{|c|}{ Biomasa aérea } & \multirow{3}{*}{$\begin{array}{l}\text { Biomasa } \\
\text { de raíces }\end{array}$} & \multirow{3}{*}{$\begin{array}{c}\text { Biomas } \\
\text { total }\end{array}$} \\
\hline & \multicolumn{2}{|c|}{ Fuste } & \multicolumn{5}{|c|}{ Сора } & \multirow{2}{*}{$\begin{array}{l}\text { Biomasa } \\
\text { aérea total }\end{array}$} & & \\
\hline & Madera & Corteza & $\begin{array}{l}\text { Ramas } \\
>7 \mathrm{~cm}\end{array}$ & $\begin{array}{l}\text { Ramas } \\
2-7 \mathrm{~cm}\end{array}$ & $\begin{array}{c}\text { Ramas } \\
0,5-2 \mathrm{~cm}\end{array}$ & $\begin{array}{c}\text { Ramas } \\
<0,5 \mathrm{~cm}\end{array}$ & Hojas & & & \\
\hline 5 & 4,878 & 0,4684 & 0 & 1,735 & 0,8978 & 0,4084 & 0,3423 & 8,730 & 2,713 & 11,44 \\
\hline 10 & 24,49 & 2,713 & 0 & 6,939 & 2,775 & 1,157 & 1,369 & 39,45 & 12,05 & 51,50 \\
\hline 15 & 62,95 & 7,580 & 0 & 15,61 & 5,369 & 2,127 & 3,080 & 96,72 & 28,82 & 125,5 \\
\hline 20 & 123,0 & 15,71 & 7,880 & 27,76 & 8,577 & 3,276 & 5,476 & 191,7 & 53,51 & 245,2 \\
\hline 25 & 206,8 & 27,66 & 43,23 & 43,37 & 12,33 & 4,581 & 8,556 & 346,5 & 86,48 & 433,0 \\
\hline 30 & 316,1 & 43,90 & 86,43 & 62,45 & 16,60 & 6,024 & 12,32 & 543,8 & 128,0 & 671,8 \\
\hline 35 & 452,5 & 64,88 & 137,5 & 85,00 & 21,33 & 7,593 & 16,77 & 785,6 & 178,3 & 963,9 \\
\hline 40 & 617,5 & 91,00 & 196,4 & 111,0 & 26,51 & 9,279 & 21,90 & 1074 & 237,7 & 1311 \\
\hline 45 & 812,4 & 122,7 & 263.2 & 140,5 & 32,11 & 11,08 & 27,72 & 1410 & 306,2 & 1716 \\
\hline 50 & 1038 & 160,2 & 337,8 & 173,5 & 38,12 & 12,97 & 34,23 & 1795 & 384,1 & 2179 \\
\hline 55 & 1296 & 203,9 & 420,3 & 209,9 & 44,52 & 14,97 & 41,41 & 2231 & 471,5 & 2703 \\
\hline 60 & 1587 & 254,3 & 510,6 & 249,8 & 51,30 & 17,06 & 49,28 & 2719 & 568,5 & 3288 \\
\hline 65 & 1912 & 311,4 & 608,8 & 293,2 & 58,43 & 19,24 & 57,84 & 3261 & 675,3 & 3936 \\
\hline 70 & 2272 & 375,8 & 714,8 & 340,0 & 65,93 & 21,51 & 67,08 & 3857 & 792,0 & 4649 \\
\hline
\end{tabular}

(1972-2009) ha sido de $760.106 \mathrm{Mg}^{2} \mathrm{n}^{-1}$ de biomasa, lo que equivale a una acumulación de carbono de 365.864 Mg año ${ }^{-1}$. La clase de diámetro que más contribuyó a la biomasa y al carbono total es la de $35 \mathrm{~cm}$, menos en el
IFN-1 donde se traslada a la clase de diámetro de $25 \mathrm{~cm}$. Los árboles de mayores dimensiones $(\geq 70 \mathrm{~cm})$ pasan de aportar un $1 \%$ a la biomasa total en el IFN-1 a un $7 \%$ en el IFN-4. 


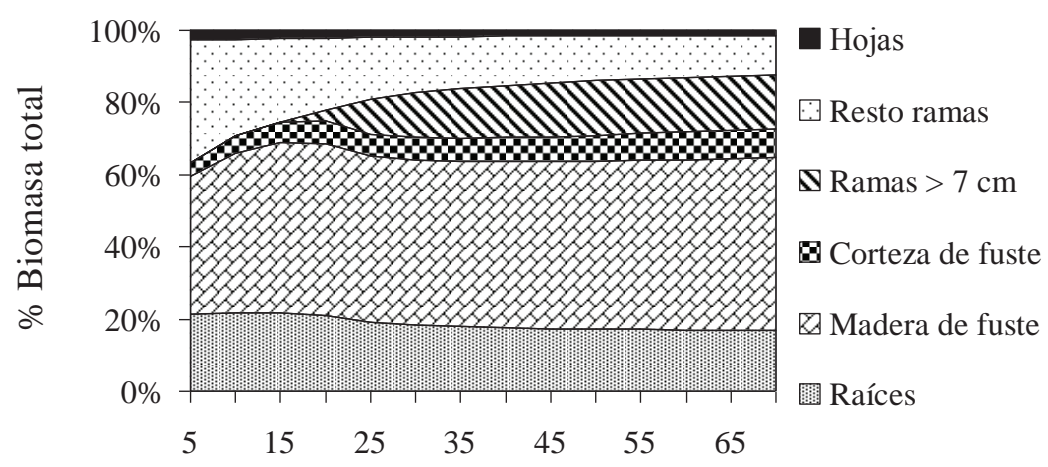

Diámetro normal (cm)

Figura 2. Aporte de cada componente a la biomasa total del árbol individual en función del diámetro normal para Q. robur en Galicia. Contribution of each component to the total biomass of individual trees in function of dbh for Q. robur in Galicia.

Cuadro 5. Contenido medio y desviación estándar (entre paréntesis) de carbono (\% p/p) en los diferentes componentes arbóreos de $Q$. robur en Galicia.

Mean concentrations (and standard deviations) of carbon (\% w/w) in different components of $Q$. robur in Galicia.

\begin{tabular}{cccccccc}
\hline \multirow{2}{*}{ Madera } & \multirow{2}{*}{ Corteza } & $\begin{array}{c}\text { Ramas } \\
>7 \mathrm{~cm}\end{array}$ & $\begin{array}{c}\text { Ramas } \\
2-7 \mathrm{~cm}\end{array}$ & $\begin{array}{c}\text { Ramas } \\
0,5-2 \mathrm{~cm}\end{array}$ & $\begin{array}{c}\text { Ramas } \\
<0,5 \mathrm{~cm}\end{array}$ & Hojas & Raíces* \\
\hline $448,1(0,5)$ & $47,6(0,9)$ & $47,6(0,6)$ & $48,0(0,5)$ & $48,5(0,4)$ & $49,2(0,5)$ & $48,6(0,6)$ & $48,6(3,9)$ \\
\hline
\end{tabular}

* Extraído de Balboa-Murias et al. (2006).

Cuadro 6. Evolución de la biomasa y del carbono acumulado por Q. robur en Galicia en los diferentes inventarios forestales nacionales (IFN). Valores en toneladas (Mg).

Changes in biomass and carbon stocks in Q. robur in Galicia in different national forest inventory (IFN). Values in tones (Mg).

\begin{tabular}{crrrrrrrr}
\hline \multirow{2}{*}{$\begin{array}{c}\text { Clase de } \\
\text { diámetro } \\
(\mathrm{cm})\end{array}$} & \multicolumn{2}{c}{ IFN-1 (año 1972) } & \multicolumn{2}{c}{ IFN-2 (año 1987) } & \multicolumn{2}{c}{ IFN-3 (año 1998) } & \multicolumn{2}{c}{ IFN-4 (año 2009) } \\
\cline { 2 - 8 } & Biomasa & Carbono & Biomasa & Carbono & Biomasa & Carbono & Biomasa & Carbono \\
\hline 5 & 678.949 & 327.718 & 980.175 & 473.116 & 2.118 .929 & 1.022 .775 & 2.144 .658 & 1.035 .195 \\
10 & 1.006 .812 & 485.653 & 1.344 .905 & 648.738 & 2.153 .385 & 1.038 .722 & 3.356 .422 & 1.619 .028 \\
15 & 1.154 .406 & 556.657 & 1.428 .529 & 688.839 & 2.327 .180 & 1.122 .170 & 3.441 .932 & 1.659 .705 \\
20 & 1.509 .426 & 727.386 & 1.719 .001 & 828.379 & 2.645 .674 & 1.274 .939 & 3.358 .278 & 1.618 .339 \\
25 & 1.800 .298 & 866.680 & 2.002 .188 & 963.871 & 2.971 .495 & 1.430 .505 & 3.490 .573 & 1.680 .394 \\
30 & 1.728 .255 & 831.600 & 2.466 .912 & 1.187 .026 & 3.342 .104 & 1.608 .150 & 3.727 .856 & 1.793 .765 \\
35 & 1.494 .925 & 719.133 & 2.602 .249 & 1.251 .810 & 3.741 .345 & 1.799 .771 & 3.957 .567 & 1.903 .785 \\
40 & 1.066 .905 & 513.147 & 2.235 .725 & 1.075 .312 & 3.235 .258 & 1.556 .055 & 3.715 .708 & 1.787 .136 \\
45 & 788.779 & 379.334 & 1.611 .049 & 774.774 & 2.517 .681 & 1.210 .785 & 3.069 .116 & 1.475 .977 \\
50 & 409.295 & 196.819 & 1.271 .836 & 611.592 & 2.229 .663 & 1.072 .185 & 2.620 .898 & 1.260 .319 \\
55 & 289.137 & 139.029 & 998.775 & 480.254 & 1.668 .455 & 802.265 & 2.174 .018 & 1.045 .362 \\
60 & 136.528 & 65.645 & 736.937 & 354.334 & 1.061 .781 & 510.525 & 1.488 .153 & 715.534 \\
65 & 42.088 & 20.236 & 546.177 & 262.602 & 885.770 & 425.879 & 828.179 & 398.190 \\
$\geq 70$ & 103.859 & 49.934 & 1.060 .483 & 509.865 & 1.849 .226 & 889.081 & 2.960 .211 & 1.423 .227 \\
\hline TOTAL & 12.209 .663 & 5.878 .971 & 21.004 .942 & 10.110 .512 & 32.747 .945 & 15.763 .807 & 40.333 .571 & 19.415 .955 \\
\hline
\end{tabular}




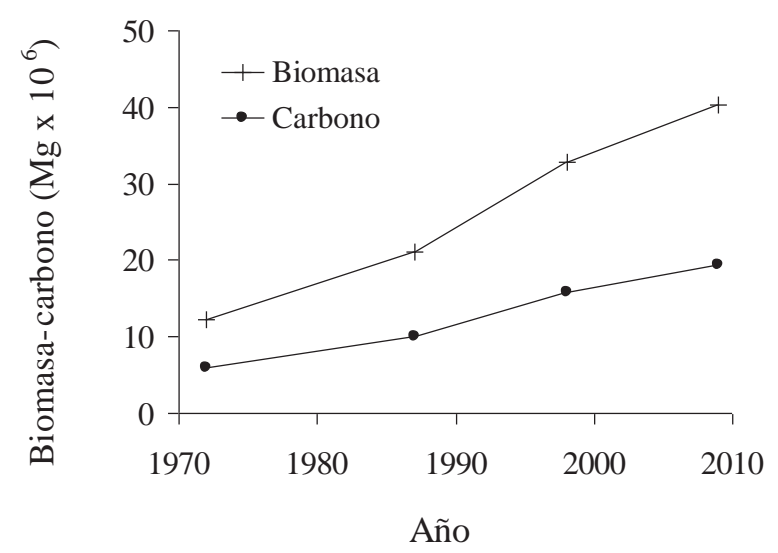

Figura 3. Evolución de la biomasa y del carbono acumulado en los árboles de $Q$. robur en Galicia.

Galicia.

Changes in biomass and carbon stocks in $Q$. robur trees in

\section{DISCUSIÓN Y CONCLUSIONES}

En este estudio se estima la evolución de la biomasa y del carbono acumulado en los árboles de Q. robur presentes en Galicia (noroeste de España). Tomando como referencia los valores modulares, los componentes de la biomasa aérea se agrupan en una proporción de biomasa de fuste que varía entre el $61 \%$ (clase de diámetro $5 \mathrm{~cm}$ ) y el 69 \% (clase de diámetro $70 \mathrm{~cm}$ ); por lo tanto la biomasa de copa varía en el intervalo 31-39\% con respecto a la biomasa aérea total. Estos resultados son acordes con los obtenidos en otros estudios para especies del mismo género en España (Rapp et al. 1999, Gracia et al. 2002) y Portugal (Carvalho y Parresol 2003), pero difieren de los obtenidos en estudios realizados en Centro Europa para Quercus petraea Matts. Liebl. (André y Ponette 2003) y en Norte-América para Q. alba L., Q. coccinea Muenchh., Q. prinus Willd. y Q. rubra L. (Martin et al. 1998), en los que la biomasa de fuste representaba un $73-84 \%$ de la biomasa aérea. Aunque, en estos dos últimos estudios, los datos procedían exclusivamente de masas mixtas. Además en el estudio de André y Ponette (2003) el rango de diámetros era diferente $(48-208 \mathrm{~cm})$ y la evolución del porcentaje de madera de fuste con respecto al total descendía al aumentar el diámetro normal, al contrario de lo que sucede en este estudio. Las raíces de los árboles de Q. robur en Galicia suponen entre un 17 y un $22 \%$ de la biomasa arbórea total; circunstancia que revela la importancia de incluir este componente en los estudios relativos al secuestro de carbono en los sistemas forestales (Dixon et al. 1994, Alexeyev et al. 1995).

En el IFN-4 también se realiza una estimación de biomasa y de carbono acumulado. Incluye tanto la biomasa aérea como la de raíces y no se considera la clase de diámetro $5 \mathrm{~cm}$. Se emplean las ecuaciones de biomasa del Instituto Nacional de Investigación y Tecnología Agraria y
Alimentaria (CIFOR-INIA) desarrolladas por Montero et al. (2005). Aunque en el IFN-4 se dice que estas ecuaciones calculan la biomasa de cada árbol a partir del diámetro y la altura medidas en campo, las ecuaciones desarrolladas por Montero et al. (2005) solo emplean el diámetro normal como variable independiente. Los valores para $Q$. robur en Galicia son 30.677.234 Mg de biomasa, de las cuales 15.338.618 Mg son de carbono. Esta estimación de biomasa que publica el IFN-4 difiere de la que se presenta en este estudio, donde, si no se considera la clase de diámetro $5 \mathrm{~cm}$, es de 38.188.913 Mg. Se considera que las ecuaciones de biomasa desarrolladas en este estudio son más representativas para Galicia que las desarrolladas por Montero et al. (2005), ya que se amplió la base de datos y su representatividad geográfica, además de disponer de observaciones de la fracción hojas y de utilizar una metodología que garantiza la aditividad de las ecuaciones.

Siendo estrictos con el Protocolo de Kyoto, no se podría imputar como sumidero todo el almacenamiento de carbono en esta especie. En su artículo 3.3 este protocolo establece que "las variaciones netas de las emisiones por las fuentes y la absorción por los sumideros de gases de efecto invernadero que se deban a la actividad humana directamente relacionada con el cambio de uso de la tierra y la silvicultura, limitada a la forestación, reforestación y deforestación desde 1990, calculadas como variaciones verificables del carbono almacenado en cada período de compromiso, serán utilizadas para los efectos de cumplir los compromisos de cada parte ...”. El aumento de las existencias de $Q$. robur en Galicia se debe principalmente a regeneración natural, por lo tanto no se podría restar la totalidad de la estimación de carbono acumulado a las emisiones, con el objetivo de cumplir los compromisos acordados. El inventario de emisiones de gases de efecto invernadero en Galicia (Xunta de Galicia 2004) estima que se reforestaron anualmente una media de 695 ha con Quercus (no se especifica la especie) en el periodo 1990-2002, con una fijación de carbono de $0,52 \mathrm{Mg} \mathrm{ha}^{-1} \mathrm{año}^{-1}$. Esto supone 32.887 Mg de carbono acumulado frente a las 5.439.541 Mg estimadas para el mismo periodo en este estudio.

Para estimar las reservas totales de carbono en los bosques de esta especie en Galicia sería necesario estimar también la biomasa y el carbono presente en la vegetación acompañante, además del carbono almacenado en la hojarasca y en el suelo mineral. Según Whittaker y Likens (1973) la hojarasca y otros tejidos muertos depositados sobre el suelo suponen un 6-8 \% de la biomasa vegetal viva. Vande Walle et al. (2001) y Balboa-Murias et al. (2006) indican que el carbono almacenado en la biomasa arbórea (aérea y subterránea) es el 50 \% del carbono acumulado en todo el sistema para bosques de $Q$. robur y Fagus sylvati$c a$ L. presentes en Bélgica y para bosques maduros de $Q$. robur presentes en Galicia, respectivamente. Fernandez et al. (2012) indican que en la capa superior del suelo (0-15 $\mathrm{cm}$ ) de bosques maduros de $Q$. robur en Galicia se acumula más de $80 \mathrm{Mg} \mathrm{ha}^{-1}$ de carbono. Aunque son estudios 
basados en un número pequeño de parcelas de muestreo y centrados en bosques adultos, dan una idea de la importancia de estos componentes.

En el desarrollo de las ecuaciones de estimación de biomasa no se incluyeron árboles que estuvieran próximos a claros o bordes de bosque o camino. Un posible estudio sería evaluar si en aquellos paisajes muy fraccionados, como puede ser Galicia, los desequilibrios de los árboles de borde modificarían significativamente la estimación de la biomasa acumulada en sus bosques. Por último, señalar que las ecuaciones de biomasa desarrolladas en este estudio también pueden utilizarse como base para estimar otras variables, además del carbono, como por ejemplo el contenido total en nutrientes para cada árbol.

\section{AGRADECIMIENTOS}

Los autores agradecen las sugerencias del Dr. Juan Gabriel Álvarez González.

\section{REFERENCIAS}

Alexeyev V, R Birdsey, V Stakanov, I Korotkov. 1995. Carbon in vegetation of Russian forests: methods to estimate storage and geographical distribution. Water, Air, and Soil Pollution 82: 271-282.

André F, Q Ponette. 2003. Comparison of biomass and nutrient content between oak (Quercus petraea) and hornbeam (Carpinus betulus) trees in a coppice-with-standards in Chimay (Belgium). Annals of Forest Science 60: 489-502.

Balboa-Murias MA, A Rojo, JG Álvarez-González, A Merino. 2006. Carbon and nutrient stocks in mature Quercus robur L. stands in NW Spain. Annals of Forest Science 63: 557565.

Belsley DA. 1991. Conditioning diagnostics, collinearity and weak data in regression. New York, USA. Wiley. 396 p.

Birdsey RA, AJ Plantinga, LS Heath. 1993. Past and prospective carbon storage in United States forest. Forest Ecology and Management 58: 33-40.

Bolin B, R Sukumar. 2000. Global perspective. In Watson RT, IR Noble, B Bolin, NH Ravindranath, DJ Verardo, DJ Dokken eds. Land Use, Land-Use Change, and Forestry: Special Report of the Intergovernmental Panel on Climate Change. Cambridge, UK. Cambridge University Press. p. 23-51.

Carvalho JP, BR Parresol. 2003. Additivity in tree components biomass of Pyrenean oak (Quercus pyrenaica Willd.). Forest Ecology and Management 179: 269-273.

Castedo-Dorado F, E Gómez-García, U Diéguez-Aranda, M Barrio-Anta, F Crecente-Campo. 2012. Aboveground standlevel biomass estimation: a comparison of two methods for major forest species in northwest Spain. Annals of Forest Science 69: 735-746.

Cohen WB, ME Harmon, DO Wallin, M Fiorella. 1996. Two decades of carbon flux from forests of the Pacific northwest. BioScience 46: 836-844.

Díaz-Fernández PM, P Jiménez Sancho, S Martín Albertos, Yde Tuero, M Reyna, L Gil Sánchez. 1995. Regiones de procedencia de Quercus robur L., Quercus petraea (Matt.) Liebl. y Quercus humillis (Miller). Madrid, España. Publicaciones del ICONA. MAPA. 87 p.

Dixon RK, S Brown, RA Houghton, AM Solomon, MC Trexler, J Wisniewski. 1994. Carbon pools and flux of global forest ecosystems. Science 263: 185-190.

Fernandez I, B Carrasco, A Cabaneiro. 2012. Evolution of soil organic matter composition and edaphic carbon effluxes following oak forest clearing for pasture: climate change implications. European Journal of Forest Research 131(6): 1681-1693.

Gil MV, D Blanco, MT Carballo, LF Calvo. 2011. Carbon stock estimates for forests in the Castilla y León region, Spain. A GIS based method for evaluating spatial distribution of residual biomass for bio-energy. Biomass and Bioenergy 35: 243-252.

Gómez-García E, F Crecente-Campo, U Diéguez-Aranda. 2013. Tarifas de biomasa aérea para abedul (Betula pubescens Ehrh.) y roble (Quercus robur L.) en el noroeste de España. Madera y Bosques 19(1): 71-91.

Gracia C, S Sabaté, J Vayreda, J Ibáñez. 2002. Aboveground Biomass Expansion Factors and biomass equations of forest in Catalonia. In Cost E21 BEF meeting. Besalú, España. 4-5 julio.

Greene WH. 2000. Econometric analysis (4th ed.). Upper Saddle River, New Jersey, USA. Prentice Hall. 1004 p.

Grigal DF, LF Ohmann. 1992. Carbon storage in upland forest of the lake states. Soil Science Society of America Journal 56: 935-943.

Huet S, F Forgeard, C Nys. 2004. Above- and belowground distribution of dry matter and carbon biomass of Atlantic beech (Fagus sylvatica L.) in a time sequence. Annals of Forest Science 61: 683-694.

Isaev A, G Korovin, D Zamolodchikov, A Utkin, A Pryaznikov. 1995. Carbon stock and deposition in phytomass of the Russian forests. Water, Air, and Soil Pollution 82: 247-256.

Jalkanen A, R Mäkipää, G Ståhl, A Lehtonen, H Petersson. 2005. Estimation of biomass stock of trees in Sweden: comparison of biomass equations and age-dependent biomass expansion factors. Annals of Forest Science 62: 845-851.

Kozak A. 1970. Methods of ensuring additivity of biomass components by regression analysis. Forestry Chronicle 46: 402404.

MARM (Ministerio de Agricultura, Alimentación y Medio Ambiente, ES). 2011. Cuarto Inventario Forestal Nacional Galicia. Madrid, España. Dirección General de Medio Natural y Política Forestal. Ministerio de Medio Ambiente, y Medio Rural y Marino. 49 p. + 4 DVD.

Martin JG, BD Kloeppel, TL Schaefer, DL Kimbler, SG Mcnulty. 1998. Aboveground biomass and nitrogen allocation of ten deciduous southern Appalachian tree species. Canadian Journal of Forest Research 28: 1648-1659.

Montero G, R Ruiz-Peinado, M Muñoz. 2005. Producción de biomasa y fijación de $\mathrm{CO}_{2}$ por los bosques españoles. Madrid, España. Monografías INIA, Serie Forestal 13. Instituto Nacional de Investigación y Tecnología Agraria y Alimentaria, Ministerio de Educación y Ciencia. 270 p.

Parresol BR. 1999. Assessing tree and stand biomass: a review with examples and critical comparisons. Forest Science 45: 573-593.

Rapp M, I Santa Regina, M Rico, HA Gallego. 1999. Biomass, nutrient content, litterfall and nutrient return to the soil in Mediterranean oak forest. Forest Ecology and Management 


\section{9: 39-49.}

Ruiz-Peinado R, G Montero, M del Río. 2012. Biomass models to estimate carbon stocks for hardwood tree species. Forest Systems 21(1): 42-52.

SAS Institute Inc. 2009. SAS/STAT ${ }^{\circledR} 9.2$ User’s Guide (2nd ed.). Cary, NC, USA. SAS Institute Inc. 7869 p.

Schoene D. 2002. Assessing and reporting forest carbon stock changes for FRA, UNFCCC and Kyoto Protocol: a concerted effort? In Luhtala A, J Varjo eds. Proceedings of FAO expert consultation on global forest resource assessment
2002. Kotka, Finland. p. 290-7.

Vande Walle I, S Mussche, R Samson, N Lust, R Lemeur. 2001. The above-and belowground carbon pools of two mixed deciduous forest stands located in East-Flanders (Belgium). Annals of Forest Science 58: 507-517.

Whittaker RH, GE Likens. 1973. Primary productivity: the biosphere and man. Human Ecology 1(4): 357-369.

Xunta de Galicia. 2004. Inventario de emisións de gases de efecto invernadoiro de Galicia. Santiago de Compostela, España. Consellería de Medio Ambiente. 167 p.

Recibido: 23.09.14

Aceptado: 13.03 .15 